\title{
BMJ Open Cross-sectional associations of sedentary behaviour and physical activity on depression in Japanese older adults: an isotemporal substitution approach
}

\author{
Akitomo Yasunaga, ${ }^{1}$ Ai Shibata, ${ }^{2}$ Kaori Ishii, ${ }^{3}$ Mohammad Javad Koohsari, ${ }^{3,4,5}$ \\ Koichiro Oka ${ }^{3}$
}

To cite: Yasunaga A, Shibata A, Ishii $\mathrm{K}$, et al. Cross-sectional associations of sedentary behaviour and physical activity on depression in Japanese older adults: an isotemporal substitution approach. BMJ Open 2018;8:e022282. doi:10.1136/ bmjopen-2018-022282

- Prepublication history for this paper is available online. To view these files, please visit the journal online (http://dx.doi org/10.1136/bmjopen-2018022282).

Received 13 February 2018 Revised 23 July 2018 Accepted 30 July 2018

Check for updates

(C) Author(s) (or their employer(s)) 2018. Re-use permitted under CC BY-NC. No commercial re-use. See rights and permissions. Published by BMJ.

${ }^{1}$ Faculty of Liberal Arts and Sciences, Bunka Gakuen University, Shibuya-ku, Japan ${ }^{2}$ Faculty Health and Sport Sciences, University of Tsukuba, Tsukuba, Japan

${ }^{3}$ Faculty of Sport Sciences, Waseda University, Tokorozawa, Japan

${ }^{4}$ Behavioural Epidemiology Laboratory, Baker Heart and Diabetes Institute, Melbourne, Australia

${ }^{5}$ Mary MacKillop Institute for Health Research, Australian Catholic University, Melbourne, Australia

Correspondence to Dr Akitomo Yasunaga; yasunaga@bunka.ac.jp

\section{ABSTRACT}

Objectives Reducing sedentary behaviour (SB) and increasing physical activity (PA) have been shown to be associated with decreased depression. However, there are yet few studies examining the potential benefits on older adults' depression, when SB is replaced with PA. This study aimed to examine the associations of objectively assessed SB, light-intensity PA (LPA) and moderate-tovigorous PA (MVPA) with depression among a sample of Japanese older adults, and to explore impacts of substituting SB with PA on older adults' depression. Design Cross-sectional analysis.

Setting General community.

Participants A total of 276 older adults aged $65-85$ years living in Japan.

Main outcome measures Three behaviours including the average daily time spent in SB $(\leq 1.5 \mathrm{METs})$; LPA $(>1.5$ to $<3.0$ METs) and MVPA ( $\geq 3.0$ METs) per day were calculated by accelerometers. Depression was assessed using the Japanese version of the 15-item Geriatric Depression Scale (GDS-15).

Results Less SB ( $\beta=0.129,95 \% \mathrm{Cl} 0.015$ to 0.243 ) and more LPA ( $\beta=-0.138,95 \% \mathrm{Cl}-0.265$ to -0.011$)$ were found to be significantly and negatively associated with the GDS-15 score in the single-activity model. The isotemporal substitution model found that replacing only 30 min per day of SB with the same amount of LPA to be significantly and negatively associated with the GDS-15 score $(\beta=-0.131,95 \% \mathrm{Cl}-0.260$ to -0.002$)$.

Conclusions These findings indicated that substituting even small amounts of SB with LPA may contribute to less depression in older adults. Potential favourable effects can be observed for replacing only 30 min per day of SB with LPA.

\section{INTRODUCTION}

Regular physical activity (PA), especially moderate-to-vigorous PA (MVPA) (eg, exercise, sports and brisk walking), has been found to be associated with less depression among older adults. ${ }^{12}$ Recent studies have also demonstrated that sedentary behaviour (SB) (eg, television viewing, computer use, and sitting in cars) and light-intensity PA
Strengths and limitations of this study

- The use of accelerometers to objectively measure participants' sedentary and active behaviours was the main strength of this study.

- We examined the impacts of substituting sedentary behaviour with physical activity on older adults' depression by using the isotemporal substitution approach.

- As a cross-sectional study, we were unable to infer a cause-and-effect relationship between sedentary behaviour, physical activity and depressive symptoms.

(LPA) (eg, housework, gardening, and casual walking) are closely related to depression: less $\mathrm{SB}^{3}$ and more $\mathrm{LPA}^{4}$ were found to be favourably associated with older adults' depression.

Several recent studies have examined how replacing one activity with another (eg, replacing SB with LPA or MVPA) can affect various health outcomes such as all-cause mortality and cardiovascular disease using the isotemporal substitution (IS) approach. ${ }^{5}{ }^{6}$ For example, a cross-sectional study found the reallocation of $30 \mathrm{~min} /$ day of SB with the equal time of sleep or LPA, or MVPA to be associated with better cardiovascular risk biomarkers. ${ }^{5}$ Another prospective study including a large sample of middle-aged and older adults found that replacing SB with the same amount of standing, sleeping (only among low sleepers), and walking or MVPA was associated with the lowest mortality risk. ${ }^{6}$ The IS approach enables researchers to simultaneously model a specific activity being performed and an activity being displaced in an equal time-exchange manner. ${ }^{6}$ There are few previous studies examining the potential benefits on depression, when SB was replaced with LPA or MVPA. ${ }^{78}$ For instance, a prospective study with 10 years follow-up among a 
large sample of US women found that replacing $60 \mathrm{~min} /$ day of television viewing time with the same amount of fast walking was associated with a lower depression. ${ }^{7}$ Another study examining association between objectively measured PA and depression demonstrated that replacing $30 \mathrm{~min} /$ day of $\mathrm{SB}$ with $\mathrm{LPA}$ was associated with a lower depression. ${ }^{8}$ However, one of these studies used self-reported measures of $\mathrm{PA}$ and $\mathrm{SB},{ }^{7}$ which are subject to recall bias (ie, lack of accuracy, validity, and reproducibility $)^{9}$ and two studies included middle-aged and older-aged people. ${ }^{78}$ Hallgren et al suggested that further research is needed to better understand the complex relationships between $\mathrm{PA}, \mathrm{SB}$, and depression. ${ }^{10}$ It is not clear yet how replacing SB with PA may affect depression among a total sample of elderly people, especially among Asian older sample.

Therefore, this study aims to examine the associations of objectively assessed SB, LPA, and MVPA with depression among a sample of Japanese older adults, and to explore impacts of substituting SB with $\mathrm{PA}$ on older adults' depression by using the IS approach.

\section{METHODS}

\section{Participants and data collection}

This study used cross-sectional data from a larger epidemiological study conducted in Matsudo city, Japan. A postal survey was sent to 3000 residents aged 65-85 years who were randomly selected from the registry of residential addresses. A total number of 349 participants (of 1250 people who responded to the postal survey) attended in a substudy, in which PA/SB was objectively calculated.

\section{Measurements}

Accelerometers (Active style Pro HJA-350IT, Omron Healthcare, Kyoto, Japan) were used to objectively measure participants' PA and SB. The detailed algorithm and validity of the accelerometer device have been described elsewhere. ${ }^{11-13}$ The device evaluates the intensity of activity by metabolic equivalents (METs) using a built-in algorithm. A previous study, in which METs for household and locomotive activities were calculated, reported a linear relationship between filtered synthetic accelerations with PA intensity. ${ }^{12}$ Participants were guided to wear the accelerometer on their waist for at least seven consecutive days-except when sleeping or during waterbased activities. To be included in the study, participants needed to wear the accelerometer for $\geq 4$ days (including one weekend day), with at least 10 hours/day of wear time each day. ${ }^{14}$ Non-wear time was defined as at least consecutive $60 \mathrm{~min}$ of 0 count value per minute $(\mathrm{cpm})$, with allowance for up to $2 \mathrm{~min}$ of some limited movement $(<50 \mathrm{cpm}) .{ }^{14}$ The daily average time spent on SB $(\leq 1.5$ METs), LIPA ( $>1.5$ to $<3.0$ METs) and MVPA ( $\geq 3.0$ METs) were calculated. These MET levels have been used by previous studies examining functional decline among older adults. ${ }^{1516}$
Depression was assessed using the Japanese language version of the 15-item Geriatric Depression Scale (GDS15). ${ }^{17}$ The GDS-15 questionnaire includes 15 questions about participants' feelings in the past week. For example, 'Do you often get bored? Yes/No'. The score ranges from 0 to 15: higher scores indicated stronger depression tendency. The GDS-15 has been widely used to assess depression symptom among older adults all over the world. In this study, the reliability of the GDS-15 (Cronbach's alpha) was 0.81 .

The following individual-level variables were considered as covariates: gender, age, body mass index, physical function, marital status, and educational attainment. Body mass index was objectively calculated by measuring the participants' height and weight. Physical function was assessed using the Japanese language version of the Medical Outcomes Survey Short Form-8 questionnaire. ${ }^{18}$

\section{Statistical analysis}

We tested the assumption that replacing SB with PA on may contribute to better older adults' depression. First, we confirmed that there are linear associations between $\mathrm{PA}, \mathrm{SB}$, and depression score; and there was also no multicollinearity between the independent variables. And then, three multiple linear regression models including a single-activity, a partition, and an IS model were conducted to examine the associations of SB, LPA, and MVPA with depression. Since a $30 \mathrm{~min}$ was used as a unit for activity, the IS models assessed the effect of replacing a $30 \mathrm{~min}$ of one activity with the equal time of another activity. Several previous studies using IS model have examined the effects of replacing a $30 \mathrm{~min}^{58}$ or a $60 \mathrm{~min}^{67}$ unit of SB with equal time of LPA or MVPA on various health outcomes. From the viewpoint of feasibility, replacing $60 \mathrm{~min}$ a day may be difficult for older adults. ${ }^{19}{ }^{20}$ Therefore, we chose the replacing $30 \mathrm{~min}$ in this study. The single-activity model analysed each activity component separately (eg, SB only) without considering the other activity types, adjusting for total wear time and covariates. The model (in the case of $\mathrm{SB})$ is shown as follows:

The GDS-15 score $=(\mathrm{b} 0) \mathrm{SB}+(\mathrm{b} 3)$ total wear time + (b4) covariates.

The partition model analysed all the activities simultaneously, without adjusting for total wear time. It is shown as below:

The GDS-15 score $=(\mathrm{b} 0)$ SB + (b1) LPA + (b2) MVPA $+(\mathrm{b} 4)$ covariates.

The coefficient for one type of activity represents the effect of increasing this type of activity while holding the other activities constant in this model. The model represents the effects of adding rather than substituting an activity type, because the total wear time is not included in the model (thus is not held constant).

The IS model assessed the effect of substituting one activity type with another for the equal amount of time (eg, replacing LPA with SB, by removing SB from the model). The IS model (in the case of omitting SB from the model) is shown as follows: 
Table 1 Characteristics of study participants

n or $M(S D), \%$

\begin{tabular}{lcc}
\hline Gender & & \\
\multicolumn{1}{l}{ Women } & 105 & $38.0 \%$ \\
\hline Age (years) & 74.4 & 5.3 \\
\hline Body mass index $\left(\mathrm{kg} / \mathrm{m}^{2}\right)$ & 23.5 & 3.2 \\
\hline Physical function & 49.7 & 5.3 \\
\hline Marital status & & \\
$\quad$ Married & 228 & $82.6 \%$ \\
\hline Educational attainment & & \\
$\quad$ University, junior college, vocational & 109 & $39.5 \%$ \\
$\quad$ school or higher level degree & & \\
\hline Total accelerometer wear time (min/day) & 902.9 & 86.7 \\
\hline SB (min/day) & 524.6 & 113.3 \\
\hline LPA (min/day) & 328.8 & 101.3 \\
\hline MVPA (min/day) & 50.0 & 32.8 \\
\hline GDS-15 score & 2.8 & 3.0 \\
\hline
\end{tabular}

SB, sedentary behaviour; LPA, light-intensity physical activity; MVPA, moderate-to-vigorous physical activity; GDS-15, 15-item Geriatric Depression Scale.

The GDS-15 score $=(\mathrm{b} 1) \mathrm{LPA}+(\mathrm{b} 2) \mathrm{MVPA}+(\mathrm{b} 3)$ total wear time $+(\mathrm{b} 4)$ covariates.

The coefficients b1 and b2 in this model represent the effect of a 30 min substitution of SB with one of the activity types (LPA or MVPA), while holding the other activity types and total wear time constant. For instance, b1 can be interpreted as the effect of replacing SB with LPA for $30 \mathrm{~min}$, while holding MVPA and total wear time constant.

All analyses were conducted using IBM SPSS Statistics V.20.0 for Windows (IBM Japan) and the level of significance was set at $\mathrm{p}<0.05$.

\section{Patient and public involvement statement}

Patients and public were not involved in developing the hypothesis, the specific aims or the research questions, nor were they involved in developing plans for design or implementation of the study.

\section{RESULTS}

Of 349 participants, data from 276 participants (171 men, 105 women) were analysed after excluding those with missing data; missing depression (4\%) and covariates $(0 \%-3.7 \%)$ and lacking valid PA accelerometer data $(12.6 \%)$. There were no significantdifferences in the rate of gender and the mean of age between analysis sample and those with missing data.

Table 1 shows the characteristics of study participants. The mean number of valid days of participant's wearing accelerometer was 7.2 days $(\mathrm{SD}=0.9)$. On average, participants wore accelerometers for 15 hours/day. The mean proportion of SB, LPA, and MVPA times to total accelerometer wearing time were $58 \%, 36 \%$, and $6 \%$, respectively. Correlation coefficients were -0.68 between SB and LPA, -0.34 between SB and MVPA, and 0.21 between LPA and MVPA. Table 2 shows the results for the single activity, partition and IS models with adjusting for covariates. The single-activity model shows that LPA was significantly and favourably associated with the GDS-15 score $(\beta=-0.138$, $\mathrm{p}<0.05$ ), and SB was significantly and positively associated with the GDS-15 score $(\beta=0.129, p<0.05)$. The partition model showed no significant associations between all activities with the GDS-15 score. The IS model showed that a 30 min unit of SB replaced with LPA to be significantly and negatively associated with the GDS-15 score $(\beta=-0.131, p<0.05)$.

\section{DISCUSSION}

This study examined how objectively measured SB, LPA and MVPA are associated with depression among Japanese older adults, and how replacing these behaviours may influence depression. Although many studies suggested that PA has favourable effects on depression in both clinical and non-clinical population, ${ }^{21-23}$ there is currently no consensus regarding the optimal amount of PA needed

Table 2 The associations of SB, LPA and MVPA with depression

\begin{tabular}{|c|c|c|c|c|c|c|}
\hline & \multicolumn{2}{|l|}{ SB } & \multicolumn{2}{|l|}{ LPA } & \multicolumn{2}{|l|}{ MVPA } \\
\hline & $\beta$ & $95 \% \mathrm{Cl}$ & $\beta$ & $95 \% \mathrm{Cl}$ & $\beta$ & $95 \% \mathrm{Cl}$ \\
\hline Single-activity model & 0.129 & $(0.015 \text { to } 0.243)^{*}$ & -0.138 & $(-0.265 \text { to }-0.011)^{\star}$ & -0.173 & $(-0.530$ to 0.184$)$ \\
\hline Partition model & 0.101 & $(-0.031$ to 0.233$)$ & -0.030 & $(-0.184$ to 0.124$)$ & -0.011 & $(-0.390$ to 0.367$)$ \\
\hline \multicolumn{7}{|l|}{ Isotemporal model } \\
\hline Replace SB with & Dropped & & -0.131 & $(-0.260 \text { to }-0.002)^{\star}$ & -0.113 & $(-0.473$ to 0.247$)$ \\
\hline Replace LPA with & 0.132 & $(0.003 \text { to } 0.261)^{*}$ & Dropped & & 0.020 & $(-0.383$ to 0.422$)$ \\
\hline Replace MVPA with & 0.118 & $(-0.242$ to 0.477$)$ & -0.013 & $(-0.395$ to 0.361$)$ & Dropped & \\
\hline
\end{tabular}

All models adjusted for gender, age, body mass index, physical function, marital status, educational attainment, and total accelerometer wear time.

$\beta=$ Regression coefficients correspond to a 30 min increment of each activity.

${ }^{*} \mathrm{P}<0.05$.

SB, sedentary behaviour; LPA, light-intensity physical activity; MVPA, moderate-to-vigorous physical activity. 
to treat depression. ${ }^{10}$ We found less SB and more LPA to be negatively associated with older adults' depression in a single-activity model (including total time held constant). Our findings are consistent with some recent studies that demonstrated the favourable effects of LPA or reducing SB on depression for older adults. ${ }^{424} 25$

In contrast with some previous studies ${ }^{26}$ we did not find any favourable effects of MVPA on depression in the three multiple linear regression models. This may be because of the age group (older adults), which was targeted in the current study. While relatively consistent effects of MVPA on depression among children and adults were reported by previous studies, ${ }^{26}$ there are mixed findings among older adults. ${ }^{27}$ For example, a randomised controlled trial study with a large sample aged 18-71 years reported that the mean reduction in depression scores were significantly larger in the physical exercise and internet-based cognitive-behavioural therapy groups compared with treatment as usual. ${ }^{28}{ }^{29}$ Furthermore, they compared the effects of different exercise intensities on post-treatment depression severity and found although there were no significant differences among light exercise, moderate exercise and vigorous exercise groups at post-treatment; the light exercise group reduced their depression score more than the moderate and vigorous exercise groups. ${ }^{30}$ Jung et al found that LPA was favourably associated with preventing depressive symptoms among older adults, but MVPA was not. ${ }^{27}$ Nevertheless, our findings showed that replacing SB with LPA had beneficial effects on older adults' depression. We found that replacing only $30 \mathrm{~min}$ of $\mathrm{SB}$ with the equal time of LPA during one day was associated with approximately $5 \%$ less depression score. Since our participants were relatively healthy, we did not consider the clinical meaning of decreasing depression score. However, a $5 \%$ reduction in depression score leads to 14 out of 62 people with a depression tendency (GDS score is over 5 point) return to normal range (GDS score is from 0 to 4 point). Currently, the evidence based for the prescription of different PA and exercise intensities for depression is weak. ${ }^{30}$ For older adults including physical frail people, however, our results may suggest that it will be effective to reduce SB time and increase LPA that accounts for a large proportion of activities in a daily life in order to decrease depression. Among older adults, it may be more practical to increase $30 \mathrm{~min}$ of LPA per day compared with MVPA.

This study has some limitations. The cause-and-effect relationship between $\mathrm{SB}, \mathrm{PA}$, and depression cannot be inferred from a cross-sectional study. In addition, our findings may not be generalisable to the Japanese population; as relatively healthy, active, and well-educated older adults participated in this study. Thus, we suggest that future intervention studies, such as randomised controlled trial studies, using a larger sample size and including frailer individuals, are needed to provide a more definitive interpretation of the present findings. Furthermore, the context and type of SB was not assessed in this study. SB consists of different types: passive sedentary time (eg, television viewing and just sitting) and mentally active sedentary time (eg, computer use and reading). ${ }^{31} 32$ Several recent studies have shown that these different types of SB may be differently associated with health outcomes including mental health. ${ }^{31}{ }^{33}$ Such a coexistence of different types of SB may play a role in the observed associations in this study. Further research is needed to explore the effects of replacing different types of SB with LPA and MVPA on depression symptoms. In addition, previous PA levels and exercise participation of participants (i.e., the amount of PA in the past 12 months) were not considered in this study. It is likely that previous amount of PA may affect the current depression status. ${ }^{34}$ The strength of this study is the use of accelerometers to objectively measure participants' SB and active behaviours.

\section{CONCLUSION}

Our findings indicated that replacing even small amounts of SB (e.g., watching television and sitting at a desk) with LPA (e.g., indoor housework and slow walking) are associated with less depression among older adults. Potential favourable effects were identified for replacing only 30 min per day of SB with LPA. These findings are useful in promoting mental health among older adults with better compliance, lower risk of injuries, and long-term sustainability.

Contributors All authors contributed equally to this work. KO: study concept and design. AY, AS, KI and KO: data analysis and interpretation and statistical analysis. AY and MJK: drafting of manuscript. AY, MJK and KO: critical revision of manuscript for intellectual content, final approval of version to be published.

Funding AY was supported by the JSPS KAKENHI Grant (\#15K01534). AS was supported the JSPS KAKENHI Grant (\#15K01647). MJK was supported by the JSPS Postdoctoral Fellowship for Research in Japan (\#17716). K0 was supported by the MEXT-Supported Program for the Strategic Research Foundation at Private Universities, 2015- 2019 the Japan Ministry of Education, Culture, Sports, Science and Technology (S1511017).

Competing interests None declared.

Patient consent Not required.

Ethics approval The study was approved by the Waseda University Institutional Committee on Human Research (2013-265) and the Institutional Review Board of Chiba Prefectural University of Health Sciences (2012-042).

Provenance and peer review Not commissioned; externally peer reviewed.

Data sharing statement No additional data sharing available.

Open access This is an open access article distributed in accordance with the Creative Commons Attribution Non Commercial (CC BY-NC 4.0) license, which permits others to distribute, remix, adapt, build upon this work non-commercially, and license their derivative works on different terms, provided the original work is properly cited, appropriate credit is given, any changes made indicated, and the use is non-commercial. See: http://creativecommons.org/licenses/by-nc/4.0/.

\section{REFERENCES}

1. Nelson ME, Rejeski WJ, Blair SN, et al. Physical activity and public health in older adults: recommendation from the American College of Sports Medicine and the American Heart Association. Circulation 2007;116:1094-105.

2. Netz Y, Wu MJ, Becker BJ, et al. Physical activity and psychological well-being in advanced age: a meta-analysis of intervention studies. Psychol Aging 2005;20:272-84. 
3. Hamer M, Stamatakis E. Prospective study of sedentary behavior, risk of depression, and cognitive impairment. Med Sci Sports Exerc 2014;46:718-23.

4. Loprinzi PD. Objectively measured light and moderate-to-vigorous physical activity is associated with lower depression levels among older US adults. Aging Ment Health 2013;17:801-5.

5. Buman MP, Winkler EA, Kurka JM, et al. Reallocating time to sleep, sedentary behaviors, or active behaviors: associations with cardiovascular disease risk biomarkers, NHANES 2005-2006. Am J Epidemiol 2014;179:323-34.

6. Stamatakis E, Rogers K, Ding D, et al. All-cause mortality effects of replacing sedentary time with physical activity and sleeping using an isotemporal substitution model: a prospective study of 201,129 midaged and older adults. Int J Behav Nutr Phys Act 2015;12:121.

7. Mekary RA, Lucas M, Pan A, et al. Isotemporal substitution analysis for physical activity, television watching, and risk of depression. Am J Epidemiol 2013;178:474-83.

8. Dillon CB, McMahon E, O'Regan G, et al. Associations between physical behaviour patterns and levels of depressive symptoms, anxiety and well-being in middle-aged adults: a cross-sectional study using isotemporal substitution models. BMJ Open 2018;8:e018978.

9. Stewart AL, Mills KM, King AC, et al. CHAMPS physical activity questionnaire for older adults: outcomes for interventions. Med Sci Sports Exerc 2001;33:1126-41.

10. Hallgren M, Herring MP, Owen N, et al. Exercise, Physical activity, and sedentary behavior in the treatment of depression: broadening the scientific perspectives and clinical opportunities. Front Psychiatry 2016;7:36.

11. Kurita S, Yano S, Ishii K, et al. Comparability of activity monitors used in Asian and Western-country studies for assessing free-living sedentary behaviour. PLoS One 2017;12:e0186523.

12. Ohkawara K, Oshima Y, Hikihara Y, et al. Real-time estimation of daily physical activity intensity by a triaxial accelerometer and a gravityremoval classification algorithm. Br J Nutr 2011;105:1681-91.

13. Oshima $\mathrm{Y}, \mathrm{Kawaguchi} \mathrm{K}$, Tanaka S, et al. Classifying household and locomotive activities using a triaxial accelerometer. Gait Posture 2010;31:370-4

14. Healy GN, Matthews CE, Dunstan DW, et al. Sedentary time and cardio-metabolic biomarkers in US adults: NHANES 2003-06. Eur Heart J 2011;32:590-7.

15. Blair CK, Morey MC, Desmond RA, et al. Light-intensity activity attenuates functional decline in older cancer survivors. Med Sci Sports Exerc 2014;46:1375-83.

16. Chmelo E, Nicklas B, Davis C, et al. Physical activity and physical function in older adults with knee osteoarthritis. J Phys Act Health 2013;10:777-83.

17. Niino N, Imaizumi T, Kawakami N. A Japanese translationof the geriatric depression scale. Clin Gerontol 1991;10:85-7.

18. Fukuhara S, Suzukamo Y. Mannual of the SF-8 Japanese Version. Kyoto: Institute for Health Outcome \& Process Evaluation Research, 2004.
19. Fitzsimons CF, Kirk A, Baker G, et al. Using an individualised consultation and activPAL ${ }^{\mathrm{TM}}$ feedback to reduce sedentary time in older Scottish adults: results of a feasibility and pilot study. Prev Med 2013;57:718-20.

20. Gardiner PA, Eakin EG, Healy GN, et al. Feasibility of reducing older adults' sedentary time. Am J Prev Med 2011;41:174-7.

21. Rebar AL, Stanton R, Geard D, et al. A meta-meta-analysis of the effect of physical activity on depression and anxiety in non-clinical adult populations. Health Psychol Rev 2015;9:366-78.

22. Cooney GM, Dwan K, Greig CA, et al. Exercise for depression. Cochrane Database Syst Rev 2013;12:CD004366.

23. Fukukawa $Y$, Nakashima C, Tsuboi S, et al. Age differences in the effect of physical activity on depressive symptoms. Psychol Aging 2004;19:346-51.

24. Tse AC, Wong TW, Lee PH. Effect of low-intensity exercise on physical and cognitive health in older adults: a systematic review. Sports Med Open 2015;1:37.

25. Zhai L, Zhang Y, Zhang D. Sedentary behaviour and the risk of depression: a meta-analysis. Br J Sports Med 2015;49:705-9.

26. Doré I, O'Loughlin JL, Beauchamp G, et al. Volume and social context of physical activity in association with mental health, anxiety and depression among youth. Prev Med 2016;91:344-50.

27. Jung S, Lee S, Lee S, et al. Relationship between physical activity levels and depressive symptoms in community-dwelling older Japanese adults. Geriatr Gerontol Int 2018;18:421-7.

28. Hallgren M, Kraepelien M, Öjehagen A, et al. Physical exercise and internet-based cognitive-behavioural therapy in the treatment of depression: randomised controlled trial. Br J Psychiatry 2015;207:227-34.

29. Hallgren M, Helgadóttir B, Herring MP, et al. Exercise and internetbased cognitive-behavioural therapy for depression: multicentre randomised controlled trial with 12-month follow-up. Br J Psychiatry 2016;209:414-20.

30. Helgadóttir B, Hallgren M, Ekblom Ö, et al. Training fast or slow? Exercise for depression: a randomized controlled trial. Prev Med 2016;91:123-31.

31. Kikuchi H, Inoue S, Sugiyama $\mathrm{T}$, et al. Distinct associations of different sedentary behaviors with health-related attributes among older adults. Prev Med 2014;67:335-9.

32. Wullems JA, Verschueren SM, Degens $\mathrm{H}$, et al. A review of the assessment and prevalence of sedentarism in older adults, its physiology/health impact and non-exercise mobility countermeasures. Biogerontology 2016;17:547-65.

33. Kesse-Guyot E, Charreire H, Andreeva VA, et al. Cross-sectional and longitudinal associations of different sedentary behaviors with cognitive performance in older adults. PLoS One 2012;7:e47831.

34. Yoshiuchi K, Nakahara R, Kumano H, et al. Yearlong physical activity and depressive symptoms in older Japanese adults: crosssectional data from the Nakanojo study. Am J Geriatr Psychiatry 2006;14:621-4. 